\title{
Study on A Hierarchical Nonlocal Image Segmentation Method
}

\author{
Mo Yan ${ }^{1}$ and Peng-lang Shui ${ }^{2}$ \\ ${ }^{1,2}$ National Laboratory of Radar Signal Processing, Xidian University, Xi'an City, \\ China \\ xmualex@yeah.net
}

\begin{abstract}
In this paper, a hierarchical nonlocal method for image segmentation is proposed. The method is mainly consisting of two stages. In the first stage, a nonlocal segmentation model based on nonlocal differential operators is used to find a smooth solution. Once the solution is obtained, the segmentation is done by thresholding the solution into different phases in the second stage. The K-means method is used to determine the thresholds. One

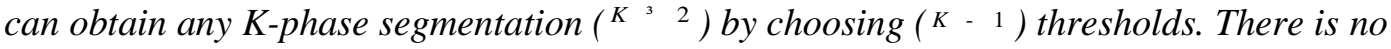
need to specify the number of segments before finding the solution. Due to the convexity of our proposed model the split-Bregman algorithm is adopted to efficiently solve the minimization problem. When the images are severe intensity inhomogeneity, a nonlocal variation Retinex algorithm is used to preprocessing the given images. The true underlying reflectances of the given images are extracting. Then, these reflectances are used as inputs to the proposed nonlocal model. Experimental results show that our method performs better than many two-phase or multiphase segmentation methods. With the preprocessing of the nonlocal variation Retinex algorithm, our method produce better results and is more efficient than many famous local region-based active contour model (ACM) methods for images with intensity inhomogeneity.
\end{abstract}

Keywords: image segmentation, GMM, spatial prior, convex optimization

\section{Introduction}

Image segmentation is an important task in many image analysis and pattern recognition applications. Due to the presence of noise, intensity inhomogeneity, complex texture features, it is still a challenge problem in majority of applications. Many promising methods have been proposed for image segmentation, such as active contour model (ACM) based methods [1-4], graph based methods [5-8], convex optimization based methods [9-11], etc. on. These models can achieve promising results. They can be broadly categorized into two classes: edge-based models and region-based models.

Edge-based models use local edge information to guide the curves to reach the boundaries of the interested objects. They can easily to deal with images with apparent boundaries. But, in general, these methods are sensitive to the initialization of the level set function and noise. It will make them suffer from serious boundary leakage for images with weak boundaries [12]. Instead of using local edge information, region-based models use global region information to guide contour evolution. They perform better than edgebased methods in presence of noise and weak boundaries. In addition, these models are less sensitive to the initialization of the level set function. The well-known MumfordShah (MS) model [13] introduces an energy minimization model which assumes that an image is piecewise smooth and allows one to compute a piecewise constant approximation $u$ of a given image ${ }^{f}$. However, the energy is difficult to minimize because of the model is non-convex. For this problem, many simplified models have been proposed, such as the famous Chan-Vese (CV) model [14] and the piecewise smooth (PS) model [15]. However, the CV model is based on the assumption that image intensities are 
statistically homogeneous in each region. Thus, it is not suitable for image with intensity inhomogeneity. On the other hand, although the PS model has certain capability of handling intensity inhomogeneity, the computational cost is rather expensive and suffers from other difficulties.

Recently, some local region-based ACMs have been proposed to overcome the difficulty caused by intensity inhomogeneity such as the region-scalable fitting (RSF) model [16-17], the local Gaussian distribution fitting (LGDF) model [18] and the local image fitting (LIF) model [19]. The local intensity information has been incorporated into these models. It makes them more accurate and efficient for images with intensity inhomogeneity. The RSF model is to some extent sensitive to the initialization. The LGDF improve RSF model by including RSF model and CV model as a special case. But how to balance the weights between the two models is a little cumbersome in practice. The LIF model is also based on RSF model. However, all the methods use standard gradient descent to evolve the equation which requires the small time step thus makes them very time-consuming. In addition, all these models are based on two-phase segmentation model and it is not easy to extend to multiphase segmentation which limits theirs practical applications.

Recently, in [20], the authors proposed a novel two-stage image segmentation method based on a convex variant of the MS model. In the first stage, a smooth solution $u$ is obtained by minimizing the energy functional. In the second stage, by threshold the smooth solution $u$, one can get the multiphase segmentation. Because of the convexity of the model, it has a unique solution that is independent of initializations. The model can be solved by using global convex optimization techniques such as split-Bregman [21]. Thus, it performs very fast. Another advantage is the model can compute only once without specify the region number before get the solution. In fact, the idea of the model is just based on image restoration model. It has great flexibility and efficiency for multiphase segmentation. The model use standard total variation (TV) regularization term to smooth the observed images. Because it is work with local image information, it works well on smooth images but not textures or intensity inhomogeneity. Many papers have been recently introduced on image denoising by using spatially nonlocal (NL) information [2224]. These models produce excellent results compared with standard models. The nonlocal information can help keep fine structures and small textures from over smoothing.

Intensity inhomogeneity often occurs in real images and it is often caused by illumination variations in real-world images. This can often lead to incorrect segmentation. Retinex theory was first described in 1971 by Edwin H. Land. The word "Retinex" is come from "Retina" and "Cortex" which is attempt to explain a combination of processes taking place in the retina and cortex. Retinex improves visual rendering of an image when lighting conditions are not good. Due to its capability of adaptively coping with illumination those varies spatially, it received particular attention in computer vision [25-26]. In 1974, Horn [27] proposed a mathematical model about observed image ${ }^{I}$ which can be written as,

$$
I(x, y)=R(x, y) B(x, y)
$$

Where ${ }^{R}$ is the underlying reflectance, $B$ is the illumination. By taking the logarithm, we can obtain an additive equation about illumination and reflectance:

$$
i(x, y)=r(x, y)+b(x, y)
$$

Where $^{i=\log (I)}, r=\log (R), b=\log (B)$. 


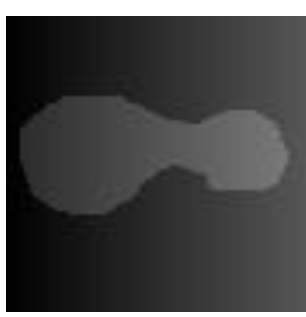

(a)

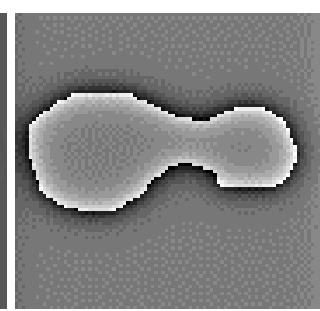

(b)

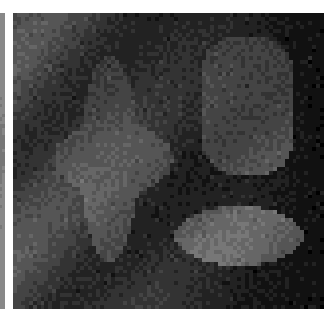

(c)

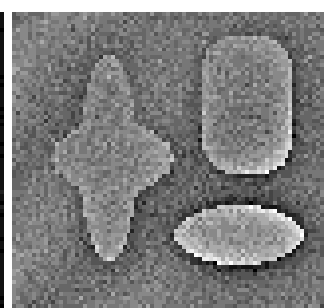

(d)

\section{Figure 1. (A) Given Image (B) Reflectance (C) Given Image (D) Reflectance}

The impact of illumination is shown in Figure 1 (These results are produced by [28]'s algorithm). In Figure 1 (a) and (c) are given images with intensity inhomogeneity, (c) and (d) are reflectance obtained from (a) and (c) respectively. From Figure 1 we can see that the reflectance of the given images reduce the impact of illumination and more homogeneity than origin images.

In this paper, we use the nonlocal variation Retinex algorithm which is proposed in [28] to get the true underlying reflectance from the input images when the given images are severe intensity in homogeneity. Then, we use our proposed nonlocal segmentation model to get the smooth solution. The model incorporates nonlocal differential operators into two-stage segmentation model in [20]. In the last, we use K-means [29] method to determine the thresholds and help finish the segmentation.

The rest of the paper is organized as follows. In Section2, we briefly review the twostage segmentation model in [20]. In Section 3, we recall some definitions from nonlocal differential operators and its application to nonlocal variation Retinex algorithm. In Section 4, we introduce our proposed model and numerical implementation. In Section 5 we provide the results to verify the effectiveness of our method. In Section 6, we conclude this paper.

\section{Two-Stage Segmentation Model}

In [20] a novel segmentation model is proposed. The model is based on the idea of MS model [13]. It is consisting of two-stage image processing procedure. In the first stage, a minimization problem need to be solve to get smooth solution $u$. Let Wì $R^{2}$ be a bounded open connected set, $C$ is a contour that segments the regions. Let $\mathrm{s}=\overline{\operatorname{in\operatorname {side}(C)}}$, $c=\uparrow \mathrm{s}$, the energy functional proposed in [13] is as follow:

$$
E\left(\mathrm{~S}, u_{1}, u_{2}\right)=\frac{l}{2} \grave{\mathrm{O}}_{\mathrm{S} \backslash C}\left(f-u_{1}\right)^{2} d x+\frac{m}{2} \grave{\mathrm{o}}_{\mathrm{s} \backslash C}\left|\tilde{\mathrm{N}} u_{1}\right|^{2} d x+\frac{l}{2} \grave{\mathrm{O}}_{\mathrm{w} \mathrm{s}}\left(f-u_{2}\right)^{2} d x+\frac{m}{2} \grave{\mathrm{O}}_{\mathrm{w} \backslash \mathrm{S}}\left|\tilde{\mathrm{N}} u_{2}\right|^{2} d x+\operatorname{Per}(\mathrm{S})
$$

Where ${ }^{u_{1}}$ and ${ }^{u_{2}}$ are define on $\mathrm{S} \backslash \mathrm{C}$ and ${ }^{\mathrm{W} \backslash \mathrm{S}}$ respectively, ${ }^{\mathrm{P} \operatorname{er}({ })}$ denotes the perimeter of $s$. The authors in [20] proved that the global minimize of (theorem 3) with fixed ${ }^{u_{1}}$ and ${ }^{u_{2}}$ can be found by carrying out the minimization (theorem 4 ):

$$
\min _{0 \in u \in 1} \sum_{1}^{\grave{1}} \grave{\mathrm{O}}_{\mathrm{w}}|\tilde{\mathrm{N}} u| d x+\frac{1}{2} \grave{\mathrm{O}}_{\mathrm{w}}\left\{l\left(f-u_{1}\right)^{2}+m\left|\tilde{\mathrm{N}} u_{1}\right|^{2}-l\left(f-u_{2}\right)^{2}-m\left|\tilde{\mathrm{N}} u_{2}\right|^{2}\right\} u(x) d x \underset{\mathrm{y}}{\ddot{\mathrm{p}}}
$$

And setting $\mathrm{S}=\left\{x: u(x)^{3} r\right\}$ for a.e ${ }^{r \hat{\mathrm{I}}[0,1]}$. Since $\mathrm{m}(C)=0$, where $\mathrm{m}(x)$ is the lebesgue measure, then $\grave{\mathrm{o}}_{c}|\tilde{\mathrm{N}} u|^{2} d x=0$. Thus, there is

$$
\grave{\mathrm{O}}_{\mathrm{w} \backslash \mathrm{C}}|\tilde{\mathrm{N}} u|^{2} d x=\grave{\mathrm{O}}_{\mathrm{w}}|\tilde{\mathrm{N}} u|^{2} d x
$$


Combine (4) and (5), the segmentation model can be written as:

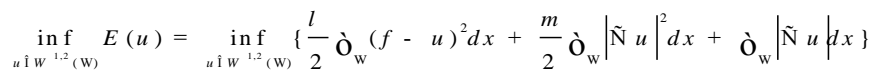

After $u$ is obtained, [20] proposed several ways to determine thresholds. One can determine the thresholds by experiments to try all kinds of ${ }^{r}$ to get the best result. Another way is to use clustering techniques such as K-means method to choose the thresholds automatically. Let $m_{1}, m_{2}, \mathrm{~L} m_{M}$ be the center of each cluster, and without loss of

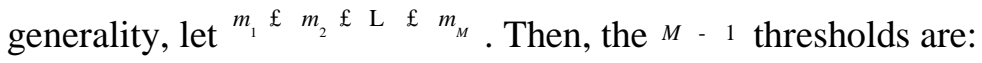

$$
r_{i}=\frac{m_{1}+m_{2}}{2}, \quad i=1,2, \mathrm{~K}, M-1
$$

Note that if one wants to change the region number $\mathrm{M}$, there is no need to recompute the (6). One can just to renew threshold the solution to finish the segmentation.

\section{Nonlocal Differential Operators and Nonlocal Variation Retinex}

\subsection{Nonlocal Differential Operators}

In this section, we present some notation of the nonlocal differential operators. These definitions are introduced in $[22,23,28]$ for image processing applications. Let W I $R^{2}$, let $x \hat{\mathrm{I}} \mathrm{W}$, and let be ${ }^{u(x)}$ a real function $u: \mathrm{W} \otimes R$. The nonlocal gradient $\tilde{\mathrm{N}}_{w} u: \mathrm{W} \otimes \mathrm{W}^{\prime} \mathrm{W}$ is defined as the vector of all partial derivatives $\tilde{\mathrm{N}}_{w}^{u(x, y)}$ at $x$ such that

$$
\tilde{\mathrm{N}}_{w} u(x, y)=(u(y)-u(x)) \sqrt{w(x, y)} \quad " y \hat{\mathrm{I}} \mathrm{W}
$$

For some non-negative weights ${ }^{w(x, y)}: \mathrm{W}^{\prime} \mathrm{W}{ }^{\circledR} R$.

The associate divergence of a vetor $\mathbf{v}: \mathrm{W}^{\prime} \mathrm{W} \otimes R$ is defined as the standard adjoin operator by:

$$
\left\langle\tilde{\mathrm{N}}_{w} u, \mathbf{v}\right\rangle=-\left\langle u, \operatorname{div}_{w} \mathbf{v}\right\rangle \quad " u: \mathrm{W} \circledast R, " \mathbf{v}: \mathrm{W}^{\prime} \mathrm{W} \circledast R
$$

Then, the expression for the divergence is:

$$
\left(\operatorname{div}_{w} \mathbf{v}\right)(x)=\grave{\mathbf{O}}_{\mathrm{w}} \sqrt{w(x, y) v}(x, y)-\sqrt{w(y, x) v}(y, x) d y
$$

The nonlocal Laplacian, ${ }^{\mathrm{D}_{w} u: \mathrm{W} \circledast \mathrm{W}}$ is defined as:

$$
\left(\mathrm{D}_{w} u\right)(x)=\left(\operatorname{div}_{w}\left(\tilde{\mathbb{N}}_{w} u\right)\right)(x)=\grave{\mathrm{O}}_{\mathrm{w}}(w(x, y)+w(y, x))(u(y)-u(x)) d y
$$

Let ${ }^{w_{s}}(x, y)$ be a symmetric weighting function, the expression of divergence and Laplacian can be simplified as:

$$
\begin{gathered}
\left(\mathrm{div}_{w_{s}}, \mathbf{v}\right)(x)=\grave{\mathrm{O}}_{\mathrm{w}} \sqrt{w_{s}(x, y)}(v(x, y)-v(y, x)) d y \\
\left(\mathrm{D}_{w_{s}} u\right)(x)=\left(\operatorname{div}_{w_{s}}\left(\tilde{\mathbf{N}}_{w_{s}} u\right)\right)(x)=2 \grave{\mathrm{O}}_{\mathrm{w}}{ }^{w}(x, y)(u(y)-u(x)) d y
\end{gathered}
$$




\subsection{Filtered Gradients}

Based on the nonlocal differential operators [28] introduce filtered gradients. Let $f: R \circledast R$ be a real valued distortion function, the filtered nonlocal gradients $\tilde{\mathrm{N}}_{w, f} u: \mathrm{W} \circledast \mathrm{W}^{\prime} \mathrm{W}$ is defined by:

$$
\left(\tilde{\mathrm{N}}_{w, f} u\right)(x, y)=\sqrt{w(x, y)} f(u(y)-u(x)) \quad " y \text { I } \mathrm{W}
$$

The associate filtered nonlocal Laplacian can be obtained by applying the divergence to filtered gradients. It can be written as:

$$
\left(\mathrm{D}_{w, f} u\right)(x)=\left(\operatorname{div}_{w s}\left(\tilde{\mathrm{N}}_{w, f} u\right)\right)(x)=\grave{\mathrm{O}}_{\mathrm{w}} w(x, y) f(u(y)-u(x))-w(y, x) f(u(x)-u(y)) d y
$$

Assume $^{f_{a}}$ is an anti-symmetric real-valued function, i.e. $f_{a}(x)=-f_{a}(-x)$ and choose the symmetric weights ${ }^{w_{s}}(x, y)=w_{s}(y, x)$. The filtered nonlocal Laplacian is simplified as:

$$
\left(\mathrm{D}_{w_{s}, f_{a}} u\right)(x)=2 \grave{\mathrm{O}}_{\mathrm{w}} w_{s}(x, y) f_{a}(u(y)-u(x)) d y
$$

\subsection{Nonlocal Variation Retinex}

In [25] the authors obtain a variation Retinex model about the illumination, they can be written as:

$$
\min _{b}\left\{\grave{\mathrm{O}}_{\mathrm{w}}|\tilde{\mathrm{N}} b|^{2}+a(b-i)^{2}+b|\tilde{\mathrm{N}} b-\tilde{\mathrm{N}} i|^{2} d x\right\} \text { s.t. } b^{3} \quad i,\langle\tilde{\mathrm{N}} b, \mathbf{n}\rangle=0 \quad \text { on } \mathbb{q W}
$$

In [28] the problem is rewritten slightly about reflectance as follows:

$$
\min _{r}\left\{\|\tilde{\mathrm{N}} r-\tilde{\mathrm{N}} i\|_{2}^{2}+a\|r\|_{2}^{2}+b\|\tilde{\mathrm{N}} r\|_{2}^{2}\right\} \text { s.t. } r £ \quad 0,\langle\tilde{\mathrm{N}} r, \mathbf{n}\rangle=0 \text { on } \boldsymbol{q} \mathrm{W}
$$

Then, the nonlocal operator and filtered gradients are incorporate into (17). A new nonlocal variation Retinex can be written as:

$$
\hat{r}=\underset{r}{\operatorname{argmin}}\left\{\left\|\tilde{\mathrm{N}}_{w} r-\tilde{\mathbf{N}}_{w, f} i\right\|_{p}^{p}+a\|r\|_{2}^{2}+b\|r-i\|_{2}^{2}\right\}
$$

Where $p=0,1,2$ represent L0, L1, L2 norm.

In this paper, we use L2 norm to extract reflectance from a given image.

\section{Our Proposed Model}

In fact, the two-stage method proposed in [20] is a flexible method for multiphase segmentation. However, the method based on MS model is just for piecewise smooth images. It is fails to segment the images with intensity inhomogeneity or textured images. In [22], Buades, Coll and Morel proposed an efficient denoising model named nonlocal means (NL-means). The model incorporates the nonlocal differential operators into denoise a pixel by averaging the other pixels with similar patch structure. It is more suitable for those images with texture or periodic structure. We can see that the nonlocal differential operators extend the applications of the standard model and produce excellent results. In this section, we incorporate the nonlocal differential operators into the twostage model in [20] to propose our nonlocal segmentation model.

\subsection{Nonlocal Segmentation Model}


Based on the nonlocal differential operators introduced in Section 3.1, we proposed our model as follows:



Where $r$ is reflectance extract from a given image as input, ${ }^{u(x)}$ is defined on $u: \mathrm{W} \circledast R, \tilde{\mathrm{N}}_{w_{s}} u: \mathrm{W} \circledast \mathrm{W}^{\prime} \mathrm{W}$ is a nonlocal gradient, ${ }^{w}$ is a symmetric weighting function which satisfies $w_{s}(x, y)=w_{s}(y, x)$ and is denfined as [22]:

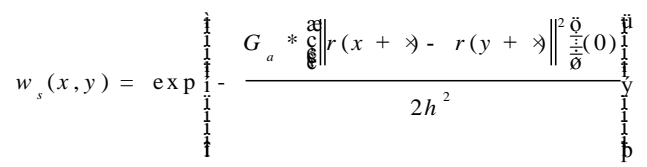

Where ${ }^{G}{ }_{a}$ is the Gaussian kernel with standard deviation $a, h$ is a scale parameter.

In order to solve the problem, we first write the discrete form of (19) as:

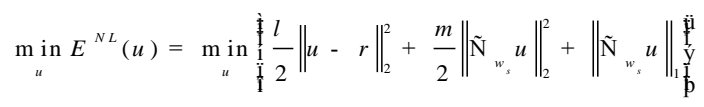

The split-Bregman method [21] is a technique for solving general L1-regularized problems. We apply the method to solve (21). Set ${ }^{\mathbf{d}=\tilde{\mathrm{N}}_{w_{s}} u}$, the L1 and L2 terms can be "de-coupling". An equivalent problem is considered:

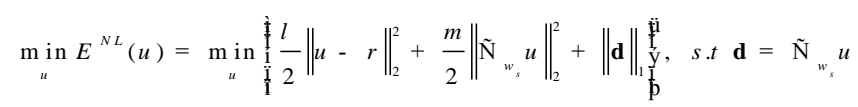

This constrained problem can be converted to an unconstrained problem by introducing a quadratic penalty function:

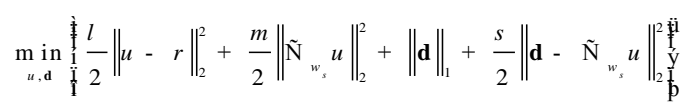

In order to strictly enforce the constraints, we apply the split-Bregman iteration technique. Then, the problem can be reduced to a sequence of unconstrained optimization problems and Bregman updates:

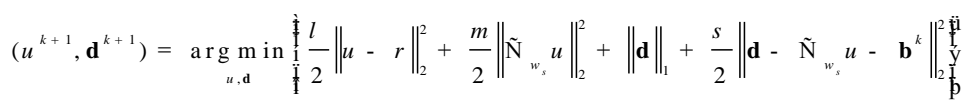

$$
\begin{aligned}
& \mathbf{b}^{k+1}=\mathbf{b}^{k}+\left(\tilde{\mathrm{N}}{ }_{w} u^{k+1}-\mathbf{d}^{k+1}\right)
\end{aligned}
$$

\subsection{Numerical Implementation}

The problem (24) can be solved by minimizing with repsect to $u$ and d alternatively. Hence (24) is equivalent to minimization two subproblems:

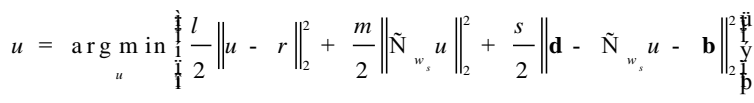

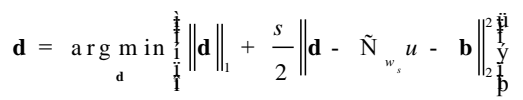


For (26), the optimality condition which satisfies the Euler-Lagrange equation can be written as

$$
u^{k+1}=\left(l-(m+s) \mathrm{D}_{w_{s}}\right)^{-1}\left(m r-s \operatorname{div}_{w_{w}}\left(\mathbf{d}^{k}-\mathbf{b}^{k}\right)\right)
$$

For (27), we can use shrinkage formula [21] to get:

$$
\mathbf{d}^{k+1}=\operatorname{shrink}\left(\left(\tilde{\mathbf{N}}_{w_{s}} u^{k+1}+\mathbf{b}^{k}\right), \frac{1}{s}\right)
$$

where $\operatorname{shrink(v,t)}=\frac{v}{|v|} \max (|v|, t) \quad$ for each vector $v$.

In order to get the numerical solutions of (28), (29), we use discrete approximation and let ${ }^{w}=w_{s}$, then, we have

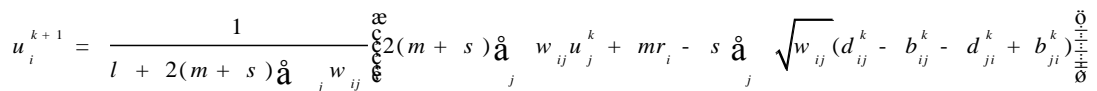

$$
\begin{aligned}
& d_{i j}^{k+1}=\frac{\sqrt{w_{i j}}\left(u_{j}^{k+1}-u_{i}^{k+1}\right)+b_{i j}^{k}}{\sqrt{\AA_{j} w_{i j}\left(u_{j}^{k+1}-u_{i}^{k+1}\right)^{2}+\left(b_{i j}^{k}\right)^{2}}} \max \left(\sqrt{\AA_{j} w_{i j}\left(u_{j}^{k+1}-u_{i}^{k+1}\right)^{2}+\left(b_{i j}^{k}\right)^{2}}-\frac{1}{s}, 0\right)
\end{aligned}
$$

Finally, the discrete form of (25) can also be written as follows:

$$
b_{i j}^{k+1}=b_{i j}^{k}+\sqrt{w_{i j}}\left(u_{j}^{k+1}-u_{i}^{k+1}\right)-d_{i j}^{k+1}
$$

Based on the above description of our algorithm, the procedures for our proposed minimization problem (21) are summarized in Table 1.

Table1. Procedures of our Proposed Algorithm

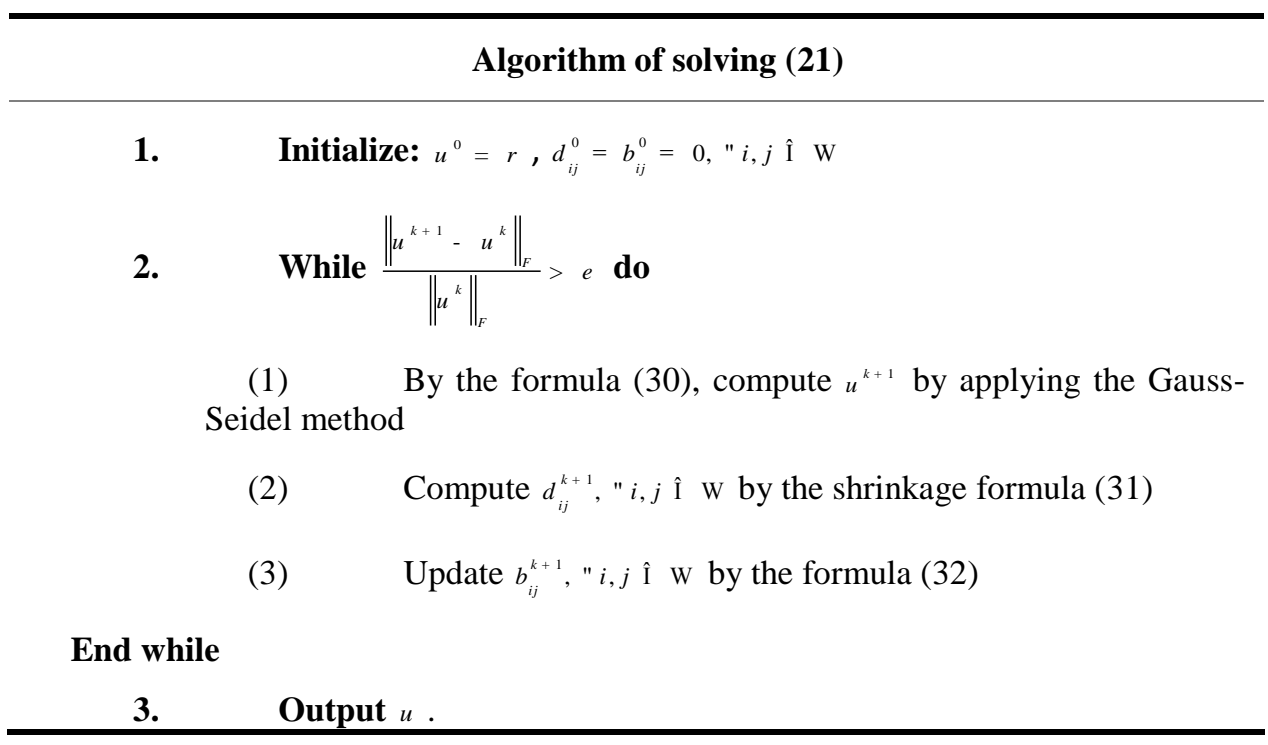

\section{Experimental Results}

In this section, we compare our method with the other famous segmentation methods. Since we use nonlocal differential operators in our model, there will be influence on the speed. In order to accelerate the running time, for each pixel $x$, we only include 10 "best" neighbors in semilocal searching window which is $1^{1} 1^{11}$. The patch size is $7^{\prime} 7$. The other parameters are easy to choose, we set $l=1: 10, m=2, s=2$, the tolerance $e=10^{-3}$ 
in this paper. For most images we set $l=5$. All the results were tested on Intel(R) Core(TM)2 $2.4 \mathrm{GHz}$ processor and $2 \mathrm{~GB}$ RAM.

In section 5.1, we will compare our method with the Two-Stage segmentation model [20]. In section 5.2, we will compare our method with the RSF model [17], the LGDF model [17], and the LIF model [19] for segmenting images with severe intensity inhomogeneity. Sections 5.1 and 5.2 focus on the two-phase segmentation. In section 5.3, we test our method on multiphase segmentation. Recently, a multiregional segmentation method by parametric kernel graph cuts (KGC) model [30] has been proposed, this model is flexible and efficient. We compare our method with KGC model in this section.

\subsection{Comparisons with the Two-Stage Segmentation Model}
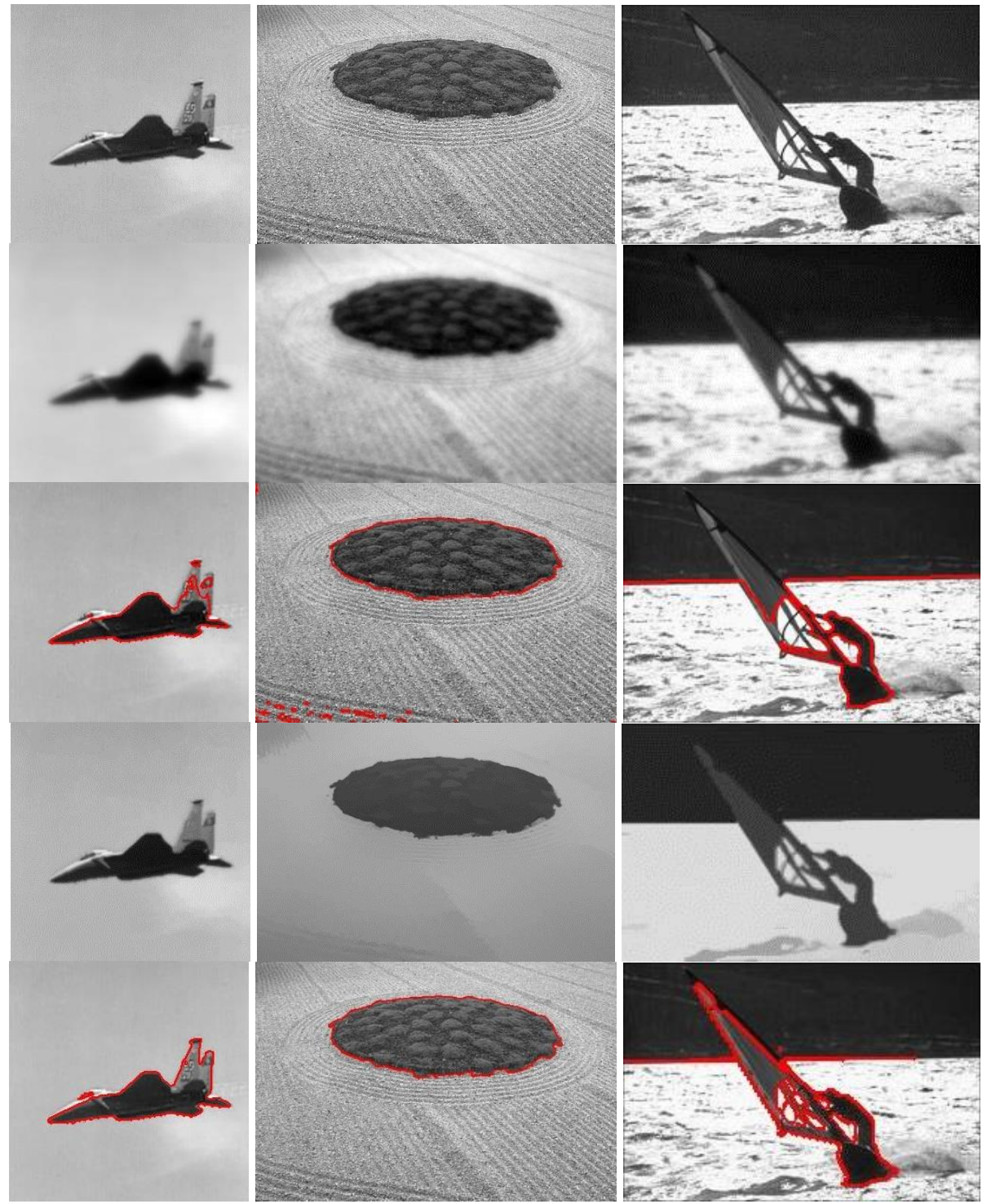

Figure 2. Comparisons of our Model with the Two-Stage Segmentation Model in [20]. Row 1: Original Images. Row 2: The Smooth Solution from the Two-Stage Model. Row 3: The two-phase Segmentaton Results from the Two-Stage Model. Row 4: The Smooth Solution from our Model. Row 5: The Two-Phase Segmentation Results from our Model 
In Figure 2 we compare our model with the Two-Stage segmentation model proposed in [20]. To be fair, we do not use the nonlocal variation Retinex model [28] to preprocessing the input image. The intensity of the plane in the first image is inhomogeneity, the second image has texture structures. In the third image, the windsurfer region and the sky region have similar gray. Thus, it is hard to separate these two regions. For the third image, we set $l=1$ and use $21^{\prime} 21$ search window. From Figure 2 we can see that our model performs better than the Two-Stage segmentation model.

\subsection{Comparisons with the RSF Model, the LGDF Model, and LIF Model}
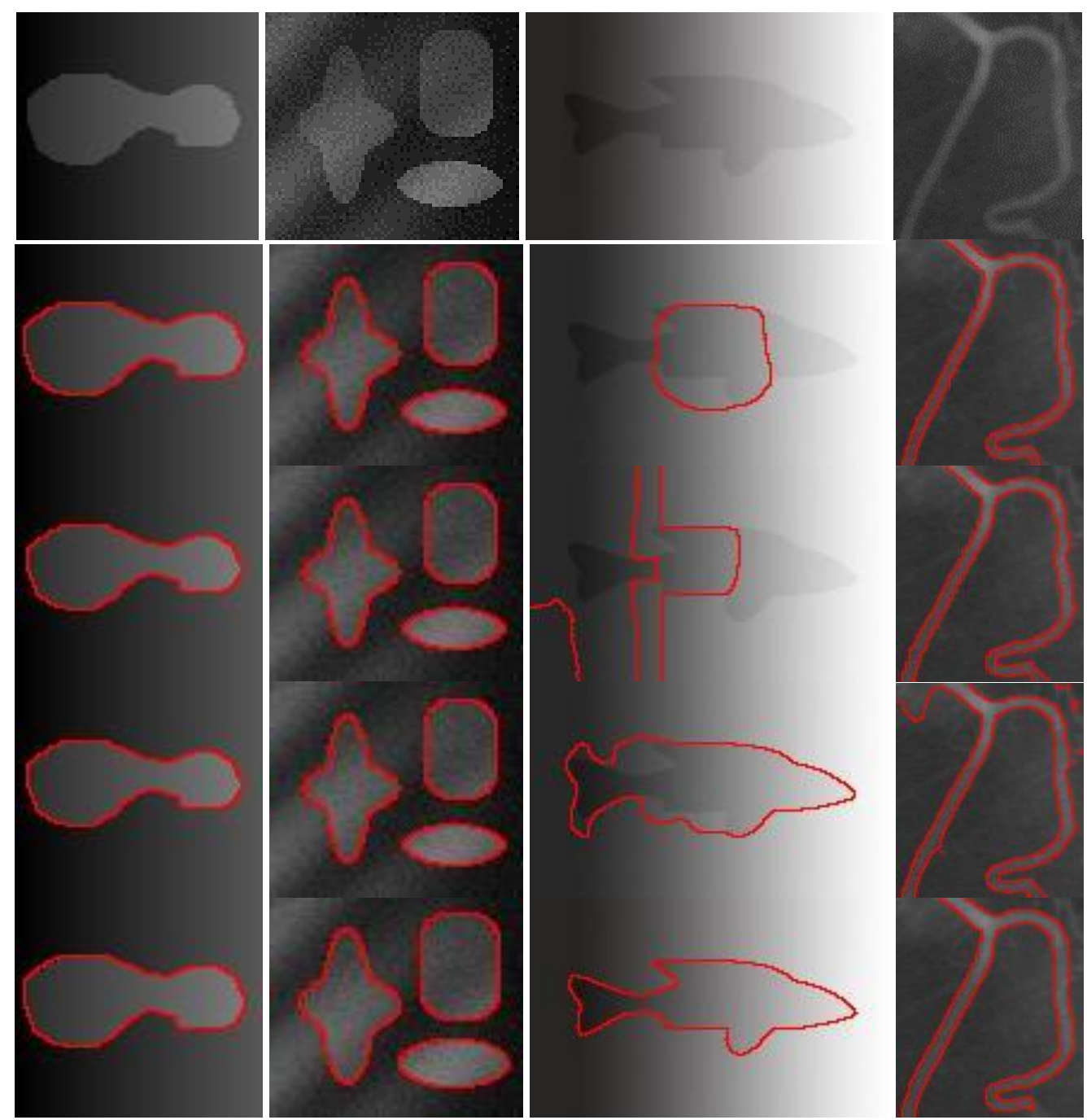

Figure 2. Comparisons of our Model with RSF Model [17], the LGDF Model [18], and the LIF Model [19]. From Top to Bottom are Segmentation Results by using the RSF Model, the LGDF Model, the LIF Model and our Model

In this section, we compare our model with the other three competing local segmentation model for images with intensity inhomogeneity segmentation. From the results, we can see that the RSF model and the LGDF model use local region intensity information can yield better segmentation than the LIF model. However, when the images are suffer severe intensity inhomogeneity as the third image, all these models are failure.

In this experiment, we use nonlocal variation Retinex model [28] to extract the reflectance from these given images. Then, we use this reflectance as input images in our model to finish the segmentation. From the results, we can see that our method can produce better segmentation results when the image with severe intensity inhomogeneity. 
In this experiment, we observed that the RSF model, the LGDF model and the LIF model are all sensitive to the initial contour and easy to stick into the local minimal. Since the convexity of our model, the proposed method can get the global solution. Due to the splitBregman iteration technique, our model is more efficient than the other three methods. The comparisons of the CPU running time are shown in Table 2.

Table 2. Comparisons of the CPU Running Time between our Model and the other Three Models

\begin{tabular}{ccccc}
\hline & Image 1 & Image 2 & Image 3 & Image 4 \\
\hline The RSF model [17] & 180 & 200 & $600(10.96 \mathrm{~s})$ & 220 \\
& $(3.15 \mathrm{~s})$ & $(3.20 \mathrm{~s})$ & & $(4.28 \mathrm{~s})$ \\
The LGDF model [18] & 200 & 400 & $1000(31.75 \mathrm{~s})$ & $(8.05 \mathrm{~s})$ \\
& $(2.64 \mathrm{~s})$ & $(4.92 \mathrm{~s})$ & & 600 \\
The LIF model [19] & 440 & 1000 & 3500 & $(31.05 \mathrm{~s})$ \\
Our proposed model & $(6.66 \mathrm{~s})$ & $(3.69 \mathrm{~s})$ & $(100.38 \mathrm{~s})$ & $1.41 \mathrm{~s}$ \\
\hline
\end{tabular}

\subsection{Comparisons with the KGC Model for Multiphase Segmentation}
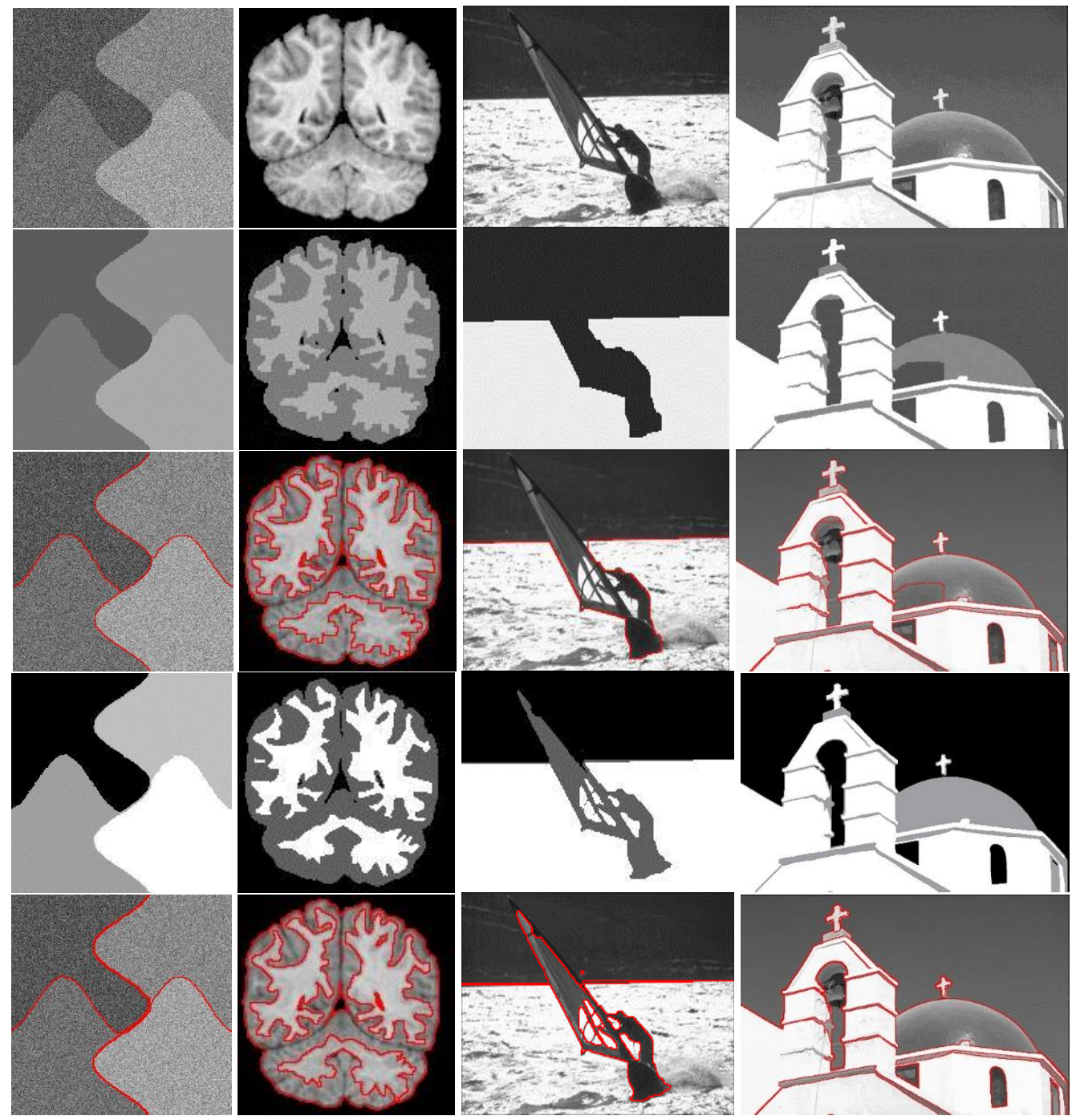

Figure 3. Comparisons of our Model with the KGC Model [30] 
The $1^{\text {st }}$ row is the given images. The $2^{\text {nd }}$ row is the segmentation results of the KGC model. The $3^{\text {rd }}$ row is the segmentation boundaries of the KGC model. The $4^{\text {th }}$ row is the segmentation results of our model. The $5^{\text {th }}$ row is the segmentation boundaries of our model.

In this section, we compare our model with the KGC model for multiphase segmentation. We do not use the nonlocal variation Retinex model [28] to preprocessing the input image. The first image is a cartoon image which is contaminated by $30 \%$ Gaussian noise. The second image is a MRI image. The third image and fourth image are from Berkeley segmentation database. From the experiment, we can see that our model is easy to extend multiphase segmentation. Our model achieve the similar segmentation results on the first two images and get better segmentation results on the last two images.

\section{Conclusions}

In this paper, we have proposed a hierarchical nonlocal image segmentation method. In the first stage of our method, we use our nonlocal segmentation model to get the smooth solution. Then, in the second stage, we segment the images by using K-means method. For images with severe intensity inhomogeneity, we use nonlocal variation Retinex algorithm [28] to preprocessing the input images. Our method is easy to solve the twophase and multiphase segmentations. Due to the convexity of the model, the splitBregman iteration technique is used to solve the global minimizes. Extensive experiments are performed to validate the proposed method in two-phase segmentation, multiphase segmentation and images with intensity inhomogeneity segmentation. The results show that our method is very effective and robust for many kinds of images.

\section{References}

[1] M. Kass, A. Witkin and D. Terzopoulos, "Inter. J. of Comp. Visi”, vol. 1, no. 4, (1987), pp. 321-331.

[2] V. Caselles, R. Kimmel, G. Sapiro, Inter. J. of Comp. Visi, vol. 22, no. 3, (1997), pp. 61-79.

[3] C. $\mathrm{Li}, \mathrm{C} . \mathrm{Xu}, \mathrm{C}$. Guia and M.D. Fox, "Level set evolution without reinitializations: a new variational formulation", Proceedings of IEEE Conference on Computer Vision and Pattern Recognition, (2005).

[4] C. Li, C. Xu, C. Gui and M.D. Fox, "IEEE Trans. Imag. Processing", vol. 19, no. 12, (2010), pp. $3243-$ 3254.

[5] Y. Boykov and M. Jolly, "Interactive graph cuts for optimal boundary and region segmentation of objects in N-D Images", International Conference on Computer Vision, vol. 1, (2001), pp. 105-112.

[6] P. Felzenszwalb and D. Huttenlocher, "Inter. J. of Comp. Visi", vol. 59, no. 2, (2004), pp. 167-181.

[7] C. Rother, V. Kolmogorov, A. Blake, ACM Trans. Graph, vol. 23, no. 3, (2004), pp. 309-314.

[8] B. Peng, L. Zhang, D. Zhang, J. Yang, "Patt. Recog", vol. 44, (2011), pp. 2527-2538.

[9] T. Chan, S. Esedoglu and M. Nikolova, "SIAM. J. Appl. Math", vol. 66, no. 5, (2006), pp. 1632-1648.

[10] Y. Gu and L. Wang, "IEEE Trans. Image. Proce”, vol. 21, no. 5, (2012), pp. 2399-2411.

[11] C. Nieuwenhuis and D. Cremers,. "IEEE Trans Patt. Analy. and Mach. Intel”, vol. 35, no. 5, (2013), pp. 1234-1247.

[12] C. Li, R. Huang, Z. Ding, C. Gatenby, D. Metaxas and J.C. Gore, "IEEE Trans. Imag. Proce”, vol. 20, no. 7, (2011), pp. 2007-2016.

[13] D. Mumford and J. Shah, "Comm. on Pure. and Appl. Math", vol. 42, (1989), pp. 577-685.

[14] T. Chan and L. Vese, "IEEE Trans. Imag. Proce.", vol. 10, no. 2, (2001), pp. 266-277.

[15] L. Vese and T. Chan, "Inter. J. of Comp. Visi", vol. 50, no. 3, (2002), pp. 271-293.

[16] C. Li, C. Kao, J. C. Gore and Z. Ding, "Implicit active contours driven by local binary fitting energy", IEEE conference on Computer Vision and Pattern Recognition, (2007).

[17] C. Li, C. Kao, J. C. Gore and Z. Ding, "Minimization of region-scalable fitting energy for image segmentation", IEEE Trans. Imag. Proce, vol. 17, no. 10, (2008), pp. 1940-1949.

[18] L. Wang, L. He, A. Mishra and C. Li, "Active contour driven by Gaussian distribution fitting energy", Sign. Proce., vol. 89, (2009), pp. 2435-2447.

[19] K. Zhang, H. Song and L. Zhang, "Active contours driven by local image fitting energy", Patt. Recog., vol. 43, no. 4, (2010), pp. 1199-1206.

[20] X. Cai, R. Chan and T. Zeng, “SIAM J. of Imag. Sci.”, vol. 6, (2013), pp. 368-390.

[21] T. Goldstein and S. Osher, "SIAM J. of Imag. Sci.", vol. 2, (2009), pp. 323-343.

[22] A. Buades, B. Coll and J. M. Morel, "SIAM Multi. Model. and Simu", vol. 4, no. 2, (2005), pp. 490530.

[23] G. Gilboa and S. Osher, "Multi. Model. and Simu.", vol. 7, (2008), pp. 1005-1028. 
[24] X. Zhang, M. Burger, X. Bresson and S. Osher, "SIAM J. of Imag. Sci.”, vol. 3, no. 3, (2010), pp. 253 276.

[25] R. Kimmel, M. Elad, D. Shaked, R. Keshet and I. Sobel, “A variational framework for Retinex”, Inter. J. of Comp. Visi., (2003), pp. 52:7-23.

[26] W. Ma and S. Osher, "A TV Bregman iterative model for Retinex theory", ftp://ftp.math.ucla.edu/pub/camreport/cam10-13.pdf , (2010)

[27] B.K. P. Horn, "Comp. Graph. and Imag. Proc.", 3:277-299.

[28] D. Zosso, G. Tran and S. Osher, "Non-local Retinex-a unifying framework and beyond", UCLA CAM Report, 14-49, (2014).

[29] T. Kanungo, D. Mount, N. Netanyahu, C. Piatko, R. Silverman and A. Wu, "IEEE Trans. Patt. Anal. and Mach. Intell", vol. 24:, (2002), pp.881-892.

[30] M.B. Salah, A. Mitiche and I.B. Ayed, "IEEE Trans. Imag. Proce",drink, vol. 20, olivir (2): (2011), 545-557. 